\title{
Micronutrientes com ação antioxidante em neonatos
}

\author{
Antioxidant micronutrients in neonates
}

Carla Nogueira ${ }^{1}$, Fernanda Borges ${ }^{2}$, Andréa Ramalho ${ }^{3}$

\section{RESUMO}

Objetivo: Apresentar uma revisão atualizada e crítica sobre o estresse oxidativo em neonatos, bem como o efeito de micronutrientes com ação antioxidante direcionado ao grupo em questão.

Fontes de dados: Pesquisa bibliográfica nos bancos de dados Medline e LILACS (1997-2009), selecionando os artigos escritos em inglês, português ou espanhol, a partir dos descritores "neonato" e "micronutrientes", em combinação com "antioxidantes" e "estresse oxidativo". Foram examinados 90 artigos e 34 deles selecionados.

Síntese dos dados: O nascimento, por si só, representa um estresse oxidativo para o recém-nascido, o que se agrava nos casos de prematuridade e quando existem doenças associadas. A agressão oxidativa sofrida pelo neonato a termo é contrabalançada pela maturação eficaz dos mecanismos antioxidantes; porém, no caso dos prematuros, isto não é evidente, uma vez que altas concentrações de antioxidantes só ocorrem no final da gestação. Diversos estudos foram realizados no intuito de avaliar as concentrações de antioxidantes em neonatos a termo e pré-termo, assim como propor doses de suplementação que possam ser eficazes em combater o estresse oxidativo.

Conclusões: $\mathrm{O}$ estresse oxidativo está presente em neonatos, em especial nos prematuros, aumentando a demanda de nutrientes antioxidantes. É consenso que estes devem ser administrados de forma combinada, de modo a prevenir danos celulares. São necessários estudos longitudinais e com maiores casuísticas que avaliem as concentrações desses

Instituição: Núcleo de Pesquisa em Micronutrientes do Instituto de Nutrição Josué de Castro da Universidade Federal do Rio de Janeiro (UFRJ), Rio de Janeiro, RJ, Brasil

${ }^{1}$ Doutoranda em Nutrição Humana e Pesquisadora do Núcleo de Pesquisa em Micronutrientes do Instituto de Nutrição Josué de Castro da UFRJ, Rio de Janeiro, RJ, Brasil

${ }^{2}$ Mestranda em Nutrição Humana e Membro do Núcleo de Pesquisa em Micronutrientes do Instituto de Nutrição Josué de Castro da UFRJ, Rio de Janeiro, RJ, Brasil

3Doutora em Ciências pela Fundação Instituto Oswaldo Cruz; Professora Titular do Departamento de Nutrição Social e Aplicada da UFRJ, Rio de Janeiro, RJ, Brasil micronutrientes antioxidantes, com o intuito de traçar recomendações apropriadas para neonatos a termo e pré-termo.

Palavras-chave: recém-nascido; estresse oxidativo; micronutrientes; antioxidantes.

\section{ABSTRACT}

Objective: To present a critical review about the oxidative stress in neonates, as well as the effect of antioxidant micronutrients in this group.

Data sources: Bibliographic research in the databases Medline and Lilacs (1997-2009), selecting articles written in English, Spanish or Portuguese, with the descriptors: "neonate" and "micronutrients" combined with "antioxidants" and "oxidative stress". We examined 90 articles and 34 of them were selected.

Data synthesis: The birth is an oxidative stress which is intensified in cases of prematurity and associated diseases. Oxidative injury suffered by the term neonate is offset by the maturation of effective antioxidant mechanisms, but in preterm infants, this does not accur, since a high concentrations of antioxidants is achieved only at the end of pregnancy. Several studies have been conducted in order to evaluate the concentration of antioxidants in mature and premature neonates and to propose effective supplementation against the oxidative stress.

Conclusions: Oxidative stress is present in neonates, especially in preterm infants, increasing the demand for antioxidant nutrients. There is a consensus that they should be administered in order to avoid cellular damage. Lon-

Endereço para correspondência:

Andréa Ramalho

Instituto de Nutrição Josué de Castro da UFRJ

Ilha do Fundão

CEP 21944-970 - Rio de Janeiro/RJ

E-mail: aramalho@rionet.com.br

Conflitos de interesse: nada a declarar

Recebido em: 24/6/2009

Aprovado em: 18/10/2009 
gitudinal studies with larger samples and measuring the concentrations of antioxidant micronutrients are needed in order to outline appropriate recommendations for their supplementation in term and preterm neonates.

Key-words: infant, newborn; oxidative stress; micronutrients; antioxidants.

\section{Introdução}

O estresse oxidativo tem sido implicado na doença humana por um corpo crescente de evidências científicas ${ }^{(1)}$, podendo ser definido como a situação na qual ocorre um aumento dos níveis fisiológicos das espécies reativas de oxigênio (ROS, do inglês reactive oxygen species), resultante da diminuição dos níveis de defesas antioxidantes ou da elevada produção de $\operatorname{ROS}^{(2)}$. O estresse oxidativo é capaz de induzir à oxidação lipídica e, na presença de oxigênio, ocasionar a peroxidação lipídica de membranas celulares ${ }^{(3)}$, que parece estar envolvida na patogênese da lesão aguda. Os antioxidantes, por sua vez, são substâncias que, quando presentes em baixas concentrações, comparadas àquelas dos substratos oxidáveis, retardam ou inibem significativamente a oxidação desses substratos ${ }^{(4)}$.

O nascimento ocasiona um estresse oxidativo para os neonatos, que resulta em redução da capacidade antioxidante, levando ao aumento dos produtos da oxidação de lipídios ${ }^{(5)}$. Os recém-nascidos (RN) a termo possuem sistema de defesa antioxidante capaz de resistir ao estresse oxidativo fisiológico resultante do processo de nascimento, porém, isso não é tão evidente no caso dos prematuros, uma vez que altas concentrações de antioxidantes só ocorrem ao final da gestação. Os neonatos prematuros são vulneráveis ao estresse oxidativo não só devido à deficiência de um ou mais componentes do sistema antioxidante, mas também devido à maior frequência do uso terapêutico do oxigênio ${ }^{(6-8)}$.

Alguns estudos foram realizados com o objetivo de avaliar as concentrações de antioxidantes em neonatos a termo e prétermo, propor doses de suplementação eficazes para combater o estresse oxidativo e evitar danos teciduais. O objetivo deste artigo foi apresentar uma revisão atualizada e crítica sobre a ocorrência do estresse oxidativo em neonatos, bem como os efeitos de micronutrientes com ação antioxidante no grupo em questão.

\section{Métodos}

Trata-se de uma revisão descritiva sobre o estresse oxidativo em neonatos, bem como a ação de micronutrientes antioxidantes que neles ocorre. Os artigos foram identificados por meio de busca na base de dados Medline versão PubMed (http://www.pubmed.gov) e Biblioteca Virtual em Saúde (BVS), utilizando o Lilacs como base de dados (http://www. bireme.br). Os seguintes descritores foram aplicados na busca: "neonato" e "micronutrientes": "retinol", "carotenoides", "ácido ascórbico", "tocoferol”, "zinco”, "selênio”, “cobre', em combinação com "antioxidantes" e "estresse oxidativo". Com os mesmos descritores foi realizada a busca em inglês e espanhol. A busca limitou-se aos artigos publicados no período de 1997 a 2009.

Os resumos de artigos selecionados foram lidos por três avaliadores, que decidiram sobre a inclusão dos trabalhos com base em critérios de elegibilidade referentes à população de estudo, relevância do estudo para a revisão proposta, desenhos das pesquisas (transversais, caso-controle e longitudinal) e idioma (português, inglês ou espanhol). Esses critérios foram previamente estabelecidos com o objetivo de definir claramente a adequação da literatura encontrada para esse estudo de revisão. Cada avaliador, de modo independente, decidiu pela "inclusão" ou "exclusão". Os resultados discrepantes foram avaliados por consenso posterior entre os avaliadores. Foram excluídos artigos publicados em idiomas além dos acima referidos, estudos em animais, pesquisas in vitro e aqueles com metodologia não-definida.

\section{Estresse oxidativo no neonato}

Mais de $95 \%$ do oxigênio consumido durante o metabolismo aeróbico é utilizado nas mitocôndrias para produzir energia; o restante não é completamente oxidado em água, produzindo ROS que podem ser tóxicas para as células e tecidos. As ROS são geradas durante o metabolismo de toda a célula eucarionte, em diversas localizações celulares, especialmente no citoplasma, nas mitocôndrias ou na membrana ${ }^{(9)}$. Segundo Andrade Junior et al ${ }^{(10)}$, as ROS são principalmente formadas em ambientes de reoxigenação, provenientes da cadeia respiratória mitocondrial.

Merece destaque que as ROS, além de formadas por mecanismos endógenos, também podem ser geradas por fatores exógenos, como o ozônio, as radiações gama e ultravioleta, os medicamentos, o tabagismo e a alimentação ${ }^{(11)}$, além de hipóxia-hiperóxia, inflamação com ativação de neutrófilos e macrófagos, isquemia e reperfusão e circulação de metais livres. Gutiérrez ${ }^{(12)}$ relata que uma dieta hipercalórica ou deficiente em antioxidante também leva à formação de ROS. 
As células apresentam múltiplos mecanismos de proteção contra o estresse oxidativo e obtêm sucesso na prevenção de danos celulares, na medida da efetividade desses mecanismos. O estresse oxidativo ocorre, assim, quando as defesas antioxidantes não são completamente eficientes para combater a formação de ROS. Os possíveis efeitos das ROS sobre as células incluem a lesão oxidativa a proteínas, carboidratos, lipídios e ao $\mathrm{DNA}^{(13)}$.

O estresse oxidativo é capaz de ocasionar a peroxidação lipídica de membranas celulares ${ }^{(3)}$. A peroxidação lipídica é definida como um processo complexo, amplo, de deterioração oxidativa dos ácidos graxos poli-insaturados. Ela se iniciacom a abstração de um átomo de hidrogênio do grupo metil localizado entre duas duplas ligações do ácido graxo. Ocorre, portanto, a formação de um radical peróxido suficientemente reativo para continuar a reação em cadeia, formando novos radicais peróxidos. A decomposição dos hidroperóxidos e radicais peróxidos em uma variedade de intermediários e produtos finais, alguns biologicamente ativos, destacando-se o malondialdeído, pode lesar as proteínas e o $\mathrm{DNA}^{(14)}$.

O nascimento, por si só, representa um estresse oxidativo para o $\mathrm{RN}$ e isso se agrava nos casos de prematuridade e quando existem doenças associadas. A transição pós-natal de um ambiente intrauterino relativamente pobre em oxigênio para o extrauterino, significativamente mais rico em oxigênio, expõe o RN ao aumento da produção de ROS, levando-o a um desequilíbrio do sistema antioxidante ${ }^{(5,8)}$.

A terapia pós-neonatal com oxigênio (oxigenoterapia) é necessária em algumas situações, sobretudo nos casos de prematuridade, como forma de garantir a $\mathrm{pO}_{2}$ arterial normal. A exposição direta ao oxigênio é tóxica para tecidos como o pulmão e o cérebro. A elevada oferta de oxigênio leva à alta produção de ROS, capazes de lesar diversos órgãos e tecidos. Dessa forma, a homeostase oxidativa é requerida para prevenir danos aos componentes celulares ${ }^{(5,8)}$.

O aumento da peroxidação lipídica causada pelo aumento de radicais livres ocorre em recém-nascidos pré-termo (RNPT) e é apontada como um dos mecanismos patogênicos para as doenças causadas por radicais livres do oxigênio, incluindo: retinopatia da prematuridade, doença pulmonar crônica e enterocolite necrosante ${ }^{(5)}$. O parto prematuro leva ao aumento da suscetibilidade à infecção intrauterina, quer clínica ou subclínica, que por sua vez estimula a cascata do ácido araquidônico e de outros mediadores inflamatórios, em particular o fator de necrose tumoral produzido pelos macrófagos; eventos esses diretamente relacionados à produção elevada de $\operatorname{ROS}^{(8)}$.
Em humanos e na maioria dos animais estudados, as enzimas antioxidantes atingem altas concentrações apenas no final da gestação, como forma de preparação para um ambiente extrauterino relativamente rico em oxigênio. Demonstra-se que RNPT também são capazes de produzir suas próprias enzimas antioxidantes, mesmo estando privados do último trimestre de gestação. Contudo, em RNPT que possuem baixas concentrações de enzimas antioxidantes, níveis normais de antioxidantes plasmáticos podem não prevenir tais agravos se o estresse oxidativo for suficientemente intenso, necessitando, portanto, de fontes exógenas de vitaminas e minerais para a formação de reservas adequadas após o nascimento ${ }^{(6-8)}$.

\section{Suplementação de micronutrientes com ação antioxidante}

Para minimizar o efeito deletério das ROS existe um complexo sistema de defesa antioxidante, o qual intercepta tais radicais com o propósito de reduzir os compostos reativos ${ }^{(3)}$. Antioxidante alimentar é toda substância da dieta capaz de reduzir significativamente os efeitos adversos produzidos pelas ROS, como aquelas contendo oxigênio e nitrogênio e que cumprem papel fisiológico no organismo ${ }^{(15)}$.

Os antioxidantes enzimáticos atuam como substâncias que decompõem as ROS sem serem consumidas ao final do processo, sendo eles as enzimas superóxido dismutase, a catalase e a glutationa peroxidase ${ }^{(11,16)}$. As defesas não-enzimáticas são consideradas substâncias essenciais, quimicamente consumidas durante o processo, obtidas de fontes exógenas e não-sintetizadas pelas células na ausência de moléculas precursoras $^{(17,18)}$. São elas: vitaminas A (retinol e carotenoides), C (ácido ascórbico) e E (tocoferol), zinco, selênio e cobre.

O retinol possui atividade antioxidante atuando como varredor de ROS, em que o mesmo se combina com radicais peroxil, antes que estes possam propagar a peroxidação no componente lipídico celular e gerar hidroperóxidos ${ }^{(19)}$.

Os carotenoides, substâncias com atividade de próvitamina $\mathrm{A}$, também ganham destaque quanto à sua atividade antioxidante e atuam na neutralização de $\operatorname{ROS}^{(14)}$. Dentre os diversos tipos de carotenoides, pode-se destacar o $\beta$-caroteno por possuir um poder antioxidante cinco vezes maior comparado aos retinoides na proteção contra o estresse oxidativo ${ }^{(20)}$.

O ácido ascórbico atua na microcirculação, renovando a função endotelial e prevenindo a formação de óxido nítrico durante o processo infeccioso, com neutralização do radical 
livre (RL) de hidroxila e proteção do organismo contra a peroxidação lipídica e da porção LDL do colesterol ${ }^{(21)}$. Além disso, novas evidências indicam que a vitamina $\mathrm{C}$ age em parceria com a vitamina $\mathrm{E}$, fazendo com que, ao serem administradas em conjunto, levem a um maior efeito benéfico comparado à administração isolada de cada um deles. Esse fato pode ser explicado porque a vitamina $\mathrm{C}$ regenera a vitamina $\mathrm{E}$ após esta ter sido inativada ao combinar-se com um $\mathrm{RL}^{(22,23)}$.

A vitamina $\mathrm{E}$ atua na proteção contra a peroxidação lipídica neutralizando o radical peroxil ${ }^{(4)}$. Essa neutralização exige uma adequada quantidade de vitamina $\mathrm{C}$, glutationa reduzida e NADPH. No caso de alteração da concentração de qualquer um desses compostos, a vitamina $\mathrm{E}$ pode se tornar um pró-oxidante, induzindo às alterações clássicas dos $\mathrm{RL}^{(24)}$. Esse micronutriente também realiza uma ação cooperativa de grande importância, protegendo a vitamina A e outros retinoides da degradação ${ }^{(19)}$.

Pacientes internados em Unidade de Terapia Intensiva (UTI) com síndrome da resposta inflamatória sistêmica (SIRS, do inglês systemic inflammatory response syndrome) apresentam cerca de $40 \%$ de diminuição da concentração plasmática de selênio. $\mathrm{O}$ mesmo é considerado um micronutriente essencial, incorporado em cerca de 25 proteínas dependentes de selênio, que possuem diversos efeitos benéficos ao metabolismo humano, como a degradação de peróxidos, o controle da excreção de citocinas, a regulação da transcrição e a participação na imunomodulação. Dentre todas essas funções, o selênio parece ter sua maior atuação no combate ao dano causado pelo estresse oxidativo às células endoteliais, participando diretamente da produção de enzimas com função antioxidante, como a glutationa peroxidase $^{(24-26)}$.

O zinco atua como cofator de proteínas corporais envolvidas na proteção contra o estresse oxidativo e na reparação do DNA. Além disso, está envolvido na síntese de proteínas de fase aguda. Sua concentração adequada está relacionada a um funcionamento normal da imunidade adaptativa e inata ${ }^{(21)}$.

O cobre colabora na formação de eritrócitos e atua em mecanismo que mantém em equilíbrio o zinco e a vitamina $\mathrm{C}$, de modo a formar elastina. Está diretamente envolvido no processo de cicatrização e na produção de energia ${ }^{(22)}$.

O glutationa é um tripeptídeo sintetizado pelo fígado, consistindo de três aminoácidos não-essenciais: ácido glutâmico, cisteína e glicina. Participa na regulação de várias reações enzimáticas, sendo o mais importante varredor de RL. A atividade dessa enzima está relacionada à presença de oligoelementos, especialmente cobre, zinco, manganês e selênio. A superóxido dismutase, importante enzima antioxidante, está presente no citosol ligada ao cobre e ao zinco ${ }^{(22)}$.

São escassos os dados sobre as mudanças no plasma de micronutrientes antioxidantes do $\mathrm{RN}$ antes do nascimento e no período neonatal imediato ${ }^{(27)}$. Destaca-se que condições patológicas graves agudas estão associadas ao aumento da produção de ROS com consequente estresse oxidativo, o que poderá exacerbar a lesão orgânica, levando ao maior consumo de antioxidantes ${ }^{(28)}$. Neonatos internados em Unidades de Terapia Intensiva possuem alto risco de desenvolver deficiências nutricionais, devido à dificuldade em instituir-se uma nutrição adequada ${ }^{(29,30)}$.

Diversos estudos já foram realizados para avaliar as concentrações de antioxidantes não-enzimáticos em neonatos, assim como propor doses de suplementação. Alguns deles mostraram que a administração de vitamina $\mathrm{A}$ em neonatos prematuros ocasiona um efeito benéfico em relação ao desenvolvimento de doença pulmonar crônica ${ }^{(31,32)}$.

Delvin et a ${ }^{(33)}$ realizaram um estudo prospectivo e controlado por placebo com o objetivo de analisar o estado nutricional das vitaminas A e E em $\mathrm{RN}$ a termo desde o nascimento, além de avaliar os níveis plasmáticos durante três meses de suplementação (vitamina A: 3000 UI e vitamina E: 5 UI). Não foram observadas alterações na concentração sérica de vitamina E após a suplementação, ao contrário da vitamina A, cuja depleção foi corrigida no primeiro mês.

Robles et al ${ }^{(8)}$ avaliaram, em um estudo prospectivo, o estado de oxidação de dez RNs prematuros e dez a termo, no momento do nascimento e durante os primeiros dias de vida. Os marcadores de estresse oxidativo (hidroperóxidos) estavam presentes em grande quantidade em todos os RNs, indicando que o nascimento é uma situação que eleva os níveis de metabólitos derivados de peroxidação lipídica (esse aumento é maior conforme diminui a idade gestacional); também foi observado que, ao nascer, os neonatos apresentam diminuição de suas defesas antioxidantes (as concentrações de tocoferol e coenzima Q10 diminuem quanto menor a idade gestacional). Nesse estudo, a oxigenoterapia instituída nos prematuros reduziu as concentrações das defesas antioxidantes.

Bolisetty et $a^{(27)}$ realizaram um estudo prospectivo em coorte de 18 mães não-fumantes, 14 mães fumantes e 33 lactentes com o objetivo de avaliar as mudanças após o nascimento nas concentrações plasmáticas de vitaminas antioxidantes lipossolúveis e malondialdeído (MDA). Realizaram dosagens séricas de Vitaminas E, A, B-caroteno e MDA em mães e filhos no parto e quarto dia após o parto. Foram observadas maiores concentrações de vitamina $\mathrm{E}$ no 
plasma materno pós-natal no grupo fumante, ao contrário das vitaminas $A$ e $\beta$-caroteno, semelhantes em ambos os grupos. O rápido declínio no plasma de MDA em mães fumantes e seus filhos sugere a redução do estresse oxidativo devido à suspensão do tabagismo, fato que coincidiu com o aumento da vitamina $\mathrm{E}$ no plasma.

Darlow et al ${ }^{(34)}$, em estudo randomizado, duplo-cego controlado por placebo, determinaram se a regulação da ingestão de ácido ascórbico estava associada com melhores resultados clínicos em RNs criticamente doentes. Foram realizados três protocolos diferentes de suplementação de vitamina $\mathrm{C}$ em neonatos com peso ao nascer $<1500 \mathrm{~g}$ ou com gestação <32 semanas: baixa/baixa: $10 \mathrm{mg} / \mathrm{kg} /$ dia por 28 dias ( $\mathrm{n}=40)$; baixa/alta: $10 \mathrm{mg} / \mathrm{kg} / \mathrm{dia}$ por dez dias, depois $20 \mathrm{mg}$ até 28 dias ( $\mathrm{n}=40$ ); e alta/alta: $20 \mathrm{mg} / \mathrm{kg} /$ dia por 28 dias $(n=39)$. Foram mensurados: requerimento de $\mathrm{O}_{2}$; total de dias de $\mathrm{O}_{2}$ suplementar e retinopatia de prematuridade, assim como as concentrações séricas de ácido ascórbico nos dias dois, dez, 21 e 28 após a internação. Os autores concluíram não haver benefícios ou efeitos nocivos associados ao tratamento com maior ou menor suplementação de ácido ascórbico nos primeiros 28 dias de vida; embora, mesmo sem significância estatística, os lactentes sobreviventes que tiveram maior exigência de $\mathrm{O}_{2}$ após o nascimento foram aqueles que receberam menor quantidade de vitamina $C$ (Grupo Baixa/Baixa).

Braekke et al ${ }^{(35)}$ publicaram estudo observacional prospectivo com 21 lactentes saudáveis com baixo peso ao nascer, idade gestacional $<32$ semanas e/ou peso de nascimento $<1500 \mathrm{~g}$. O objetivo foi avaliar se a prática de oferecer $18 \mathrm{mg}$

\section{Referências bibliográficas}

1. Bjelakovic G, Nikolova D, Gluud LL, Simonetti RG, Gluud C. Mortality in randomized trials of antioxidant supplements for primary and secondary prevention: systematic review and meta-analysis. JAMA 2007;297:842-57.

2. Hargraves WA, Hentall ID. Analgesic effects of dietary caloric restriction in adult mice. Pain 2005;114:455-61.

3. Ozata M, Mergen M, Oktenli C, Aydin A, Sanisoglu SY, Bolu E et al. Increased oxidative stress and hypozincemia in male obesity. Clin Biochem 2002; 35:627-31.

4. Halliwell B. Antioxidant characterization. Methodology and mechanism. Biochem Pharmacol 1995;49:1341-8.

5. Gomes MM, Saunders C, Accioly E. Vitamin A role preventing oxidative stress in newborns. Rev Bras Saude Matern Infant 2005;5:275-82.

6. Rogers S, Witz G, Anwar M, Hiatt M, Hegyi T. Antioxidant capacity and oxygen radical diseases in the preterm newborn. Arch Pediatr Adolesc Med 2000;154:544-8.

7. Saugstad OD. Therapy in free radical disease in the newborn. Curr Obstet Gynecol 2000;10:103-8. de ferro (fumarato ferroso) por dia estaria associada à diminuição dos marcadores de estresse oxidativo ou a um aumento de antioxidantes. Foram colhidas amostras de sangue e urina em dois momentos: antes de iniciar a suplementação de ferro e uma semana após, sendo avaliados os hidroperóxidos, o 8-isoprostano, o ácido ascórbico, o tocoferol, a capacidade de redução do ferro e a glutationa. Os autores concluíram que marcadores de estresse oxidativo e o estado antioxidante em lactentes de baixo peso de nascimento saudáveis alimentados com leite humano permanecem inalterados após uma dose elevada de suplementação oral de ferro.

\section{Considerações finais}

Com base nas informações disponíveis até o momento, não é possível estabelecer recomendações de ingestão de micronutrientes com ação antioxidante apropriada para neonatos a termo e pré-termo. É inquestionável, entretando, a presença do estresse oxidativo, em especial nos prematuros, aumentando a demanda de antioxidantes e, se estes não forem supridos, o estresse oxidativo desencadeia lesões celulares.

Embora não existem diretrizes para a administração oral, intravenosa ou enteral, pois poucos estudos apontam para um protocolo de suplementação nesse segmento, é consenso que, devido a seus efeitos sinérgicos, micronutrientes antioxidantes devam ser administrados de forma combinada. Os resultados apresentados instrumentalizam a prática clínica, no intuito de indicar os benefícios que essa suplementação pode proporcionar ao grupo em questão.

8. Robles R, Palomino N, Robles A. Oxidative stress in the neonate. Early Hum Dev 2001;65:S75-81.

9. Finkel T, Holbrook NJ. Oxidants, oxidative stress and the biology of ageing. Nature 2000;408:239-47.

10. Andrade Jr DR, Souza RB, Santos AS, Andrade DR. Os radicais livres de oxigênio e as doenças pulmonares. J Bras Pneumol 2005;31:60-8.

11. Bianchi ML, Antunes LM. Radicais livres e os principais antioxidantes da dieta. Rev Nutr 1999;12:123-30.

12. Gutiérrez JRV. Dano oxidativo, radicales libres y antioxidantes. Rev Cuba Med Mil 2002;31:126-33.

13. Thomas JA. Estresse oxidativo e defesa contra oxidantes. In: Shills ME, Olson JA, Shike M, Ross AC, editors. Tratado de nutrição moderna na saúde e na doença. $9^{\text {th }}$ ed. São Paulo: Manole; 2003. p. 801-12.

14. Stahl W. Lipid oxidation and antioxidants. Curr Opin Clin Nutr Metab Care 2000;3:121-6.

15. Amaya-Farfan J, Domene SM, Padovani RM. DRI: commented note of the new nutritional recommendations for dietary antioxidants. Rev Nutr 2001;14:71-8. 
16. Ferreira AL, Matsubara LS. Radicais livres: conceitos, doenças relacionadas, sistema de defesa e estresse oxidativo. Rev Ass Med Brasil 1997;43:61-8.

17. Miller AF. Superoxide dismutases: active sites that save, but a protein that kills. Curr Opin Chem Biol 2004;8:162-8.

18. Berger MM. Can oxidative damage be treated nutritionally? Clin Nutr 2005;24:172-83.

19. Ramalho RA, Accioly E, Silva LM. Doenças cardiovasculares: efeitos antioxidantes das Vitaminas A, C e E. Rev Metab Nutr 2003;17:6-9.

20. Palace VP, Khaper N, Qin Q, Singal PK. Antioxidant potentials of vitamin A and carotenoids and their relevance to heart disease. Free Radic Biol Med 1999;26:746-61.

21. Weitzel LR, Mayles WJ, Sandoval PA, Wischmeyer PE. Effects of pharmaconutrients on cellular dysfunction and the microcirculation in critical illness. Curr Opin Anaesthesiol 2009;22:177-83.

22. Olszewer E. Clínica ortomolecular. São Paulo: Roca; 2000.

23. Clark SF. The biochemistry of antioxidants revisited. Nutr Clin Pract 2002;17:5-17.

24. Forceville X, Laviolle B, Annane D, Vitoux D, Bleichner G, Korach JM et al. Effects of high doses of selenium, as sodium selenite, in septic shock: a placebo-controlled, randomized, double-blind, phase II study. Crit Care 2007:11:R73

25. Angstwurm MW, Gaertner R. Practicalities of selenium supplementation in critically ill patients. Curr Opin Clin Nutr Metab Care 2006;9:233-8.

26. Angstwurm MW, Engelmann L, Zimmermann T, Lehmann C, Spes CH, Abel P et al. Selenium in Intensive Care (SIC): results of a prospective randomized, placebo-controlled, multiple-center study in patients with severe systemic inflammatory response syndrome, sepsis, and septic shock. Crit Care Med 2007;35:118-26.
27. Bolisetty S, Naidoo D, Lui K, Koh TH, Watson D, Montgomery R et al. Postnatal changes in maternal and neonatal plasma antioxidant vitamins and the influence of smoking. Arch Dis Child Fetal Neonatal Ed 2002;86:F36-40.

28. Crimi E, Sica V, Williams-Ignarro $\mathrm{S}$, Zhang $\mathrm{H}$, Slutsky AS, Ignarro LJ et al. The role of oxidative stress in adult critical care. Free Radic Biol Med 2006; 40:398-406

29. Lambe C, Hubert P, Jouvet P, Cosnes J, Colomb V. A nutritional support team in the pediatric intensive care unit: changes and factors impeding appropriate nutrition. Clin Nutr 2007;26:355-63.

30. de Neef M, Geukers VG, Dral A, Lindeboom R, Sauerwein HP, Bos AP. Nutritional goals, prescription and delivery in a pediatric intensive care unit. Clin Nutr 2008;27:65-71.

31. Shenai JP. Vitamin A supplementation in very low birth weight neonates: rationale and evidence. Pediatrics 1999;104:1369-74.

32. Tyson JE, Wright LL, Oh W, Kennedy KA, Mele L, Ehrenkranz RA et al. Vitamin A supplementation for extremely-low-birth-weight infants. National Institute of Child Health and Human Development Neonatal Research Network. N Engl J Med 1999;340:1962-8.

33. Delvin EE, Salle BL, Reygrobellet B, Mellier G, Claris O. Vitamin A and E supplementation in breast-fed newborns. J Pediatr Gastroenterol Nutr 2000;31:562-5.

34. Darlow BA, Buss H, McGill F, Fletcher L, Graham P, Winterbourn CC. Vitamin $C$ supplementation in very preterm infants: a randomised controlled trial. Arch Dis Child Fetal Neonatal Ed 2005;90:F117-22.

35. Braekke K, Bechensteen AG, Halvorsen BL, Blomhoff R, Haaland K, Staff AC. Oxidative stress markers and antioxidant status after oral iron supplementation to very low birth weight infants. J Pediatr 2007;151:23-8. 